\title{
THE IMPACT OF INCREASED BODY MASS INDEX ON SYSTEMIC LUPUS ERYTHEMATOSUS
}

Elisa Guimarães Motta ${ }^{1, *}$, Anna Beatriz Gomes Souza Duarte ${ }^{1}$, José Pinto de Souza Neto ${ }^{1}$, Gabriellen Vitiello ${ }^{1}$, Marilena Mesquita Leal Fernandes ${ }^{1}$ Mauro Goldfarb ${ }^{1}$, Adriana Danowski ${ }^{1}$

1.Hospital Federal dos Servidores do Estado, Rio de Janeiro (RJ), Brazil.

*Corresponding author: elisaguimotta@gmail.com

\section{BACKGROUND}

Studies of the impact of obesity on systemic lupus erythematosus (SLE) activity and other domains have been quite scarce. The aims of this study were to examine the impact of an increased body mass index (BMI) on damage accrual, fibromyalgia, clinical manifestations, serology and complications in a cohort of SLE.

\section{MATERIALS AND METHODS}

A cross-sectional investigation was performed. Systemic lupus erythematosus patients ( $\geq 4$ ACR revised criteria) that visited the lupus clinic for routine consultations between July of 2019 and July of 2020 were recruited by consecutive sampling. Body mass index (BMI) was used as the measurement for obesity, as the cut-off point used was $\geq 30 \mathrm{~kg} / \mathrm{m} 2$ or $25-29.9 \mathrm{~kg} / \mathrm{m} 2 \mathrm{for}$ overweight. The Systemic Lupus International Collaborating Clinics/American College of Rheumatology (SLICC/ACR) damage index (SDI) was the score used to determinate damage accrual. Univariate analyses were carried out on each potential predictive factor with Pearson's correlation or the Fisher's test as appropriate.

\section{RESULTS}

Three hundred fifty-four patients were included, 94.6\% female, 39.8\% Caucasian. The mean BMI was 28.3 kg/m2. There were $28.2 \%$ with overweight, $23.7 \%$ with obesity $9.9 \%$ with severe obesity ( $\geq 35$ ) and $3.4 \%$ with morbid obesity $(\geq 40)$ in this sample. $\mathrm{A} B \mathrm{BM} \geq 30 \mathrm{~kg} / \mathrm{m} 2$ was negatively associated with kidney chronic failure $(p=0.03)$. When applying the cut-off for overweight, the following variables were associated with BMI: high cholesterol ( $p<0.001$; odds ratio, OR $=2.76$; confidence interval, $95 \%$ $C l=1.59-4.77$ ), hypertension $(p=0.01 ; O R=1.86 ; 95 \% C l=1.15-3.00)$, Sjögren's syndrome $(p=0.07 ; O R=6.44 ; 95 \%$ $\mathrm{Cl}=1.43-28.89)$, photosensitivity $(p=0.03 ; \mathrm{OR}=1.74 ; 95 \% \mathrm{Cl}=1.03-2.91)$ and anti-RNP $(\mathrm{p}=0.03 ; \mathrm{OR}=1.74 ; 95 \% \mathrm{Cl}=1.02-2.96)$, kidney chronic failure $(p=0.04 ; O R=0.21 ; 95 \% C l=0.06-0.66)$ and hemolytic anemia $(p=0.01 ; O R=0.43 ; 95 \% C l=0.22-0.83)$. No associations were found with damage accrual, fibromyalgia or medications.

\section{CONCLUSIONS}

Obesity is a major health problem and we found a high prevalence of obesity and overweight in this sample of our SLE population. It is extremely important to take measures to reduce weight since it is significantly correlated with complications such as high cholesterol and hypertension as in the general population. We also found a cluster of patients with a higher BMI, Sjögren's syndrome and anti-RNP antibodies. No major observations could have been made on other relations. 